\title{
The adaptive capabilities of Elodea canadensis under the influence of anthropogenic factors
}

\author{
Lydia Bondareva ${ }^{1, *}$ and Galina Sorokina ${ }^{2}$ \\ ${ }^{1}$ Federal Scientific Centre of Hygiene named after F.F. Erisman, of the Russian Federal Service for \\ Surveillance on Consumer Rights Protection and Human Wellbeing, Mytischi, Moscow region, \\ Russia \\ ${ }^{2}$ Siberian Federal University, Krasnoyarsk, Russia
}

\begin{abstract}
Studies on the potential adaptation of one of the common aquatic macrophytes Elodea canadensis when immersed in a medium containing anthropogenic pollutants of various origins - metals (inorganic salts); organic salts, mineral acids; oil and its derivatives-watersoluble petrochemicals. It was found that almost all the studied pollutants do not show a clear external effect on the solid fragments of the plant (cell membranes). Thus, it was shown that Elodea canadensis is tolerant of anthropogenic pollutants that differ in nature, hazard class, physicochemical properties, etc.
\end{abstract}

\section{Introduction}

Submerged macrophytes are extremely important because they serve multiple ecological functions, such as increasing the self-purification capacity of a lake ecosystem, preventing the resuspension of sediments, reducing the release of pollutants and improving general environmental conditions [1, 2]. However, many lakes and waterways worldwide have become eutrophic, resulting in the unwanted growth of invasive aquatic vegetation. In some cases, submerged macrophytes cause silting by adding large amounts of plant material to the lake bottom and releasing pollutants to the lake water when they decay [3]. These effects indicate that the ecological functions of submerged macrophytes have been destroyed if the density of submerged macrophytes is too high.

The organism and the environment constitute a unity that is constantly thrown off balance as a result of changes in the environment and the organism itself. But almost any imbalance sooner or later is restored as a result of the adaptability of the organism to the changeable external environment. Biological systems exhibit a wide range of mechanisms and strategies to ensure their survival under variable conditions. In some cases, they compensate for internal or external perturbations by maintaining the conditions under which their constitutive processes remain viable (through physical buffering, relative damping of the changes in concentration, temperature, $\mathrm{pH} . .$.$) ; in other cases, to achieve a$ similar outcome, they switch between different available metabolic regimes (e.g., distinct

* Corresponding author: 1-bondareva@mail.ru 
biosynthetic routes linked to diauxic shifts). All these mechanisms, usually associated with the concepts of 'homeostasis' and 'adaptation' respectively, tend to be broadly interpreted as regulatory, insofar as they contribute to maintain the system's viability against perturbations by functionally modulating its own dynamic behaviour [4]. Adaptation as one of the fundamental properties of living systems, reflects, on the one hand, the resilience of biological systems to adverse environmental conditions, and on the other, the process of adaptation of living organisms to constantly changing environmental conditions. Adaptation is possible up to a certain level, which depends on the intensity and duration of exposure. Adaptation of an aquatic plant organism includes any beneficial changes at any level of the organization of the biological system (molecular, subcellular, cellular, etc.) that help its survival under changing environmental conditions.

The purpose of this research is to study the effect of anthropogenic pollutants in the aquatic environment on plant adaptation, using the example of Elodea canadensis.

\section{Objects and methods}

\subsection{Subject of study}

Aquaticm acrophyte of elodea (Elodea canadensis) was chosen as the main object of research. This type of plant is widespread in freshwater ecosystems and is quite easily cultivated under artificial conditions. The shoots of the elodea plants were harvested immediately before the series of experiments were carried out in the Yenisei River, in the area with minimal anthropogenic impact. Having been harvested, the plants were thoroughly washed with plenty of water and sorted by the quality of the biomass. Of all biomass only the apical shoots from 3 to $6 \mathrm{~cm}$ long were utilized in the experiment. Prior to the experiments, the biomass was placed in an aquarium with water, which was installed in a climate-controlled room with a constant $\mathrm{t} \sim 18^{\circ} \mathrm{C}$ and a lighting regime of $12 / 12$ hours. Experiments were carried out to verify the effects of certain organic (oil, petroleum products, organic salts) and inorganic (metals, mineral acids) pollutants. In order to determine the level of impact of anthropogenic pollutants on aquatic plants, apical whorls of Elodea were used for experiments on the sensitivity of Canadian Elodea to the action of different concentrations of heavy metal ions. Solutions of salts of nickel, copper, zinc, and lead were prepared by dissolving an exact quantity in $600 \mathrm{ml}$ of water from the Yenisei River, which was poured into the lab vessels with the addition of salt solutions with following concentrations (mg/l): $\mathrm{Co}^{2+}, \mathrm{Mn}^{2+}, \mathrm{Zn}^{2+}, \mathrm{Ni}^{2+}-0.005,0.01,0.05,0.25$; $\mathrm{Cd}^{2+}$ 0.001, 0.005, 0.025, 0.125; $\mathrm{Cu}^{2+}-0.0005,0.001,0.005,0.025, \mathrm{Al}^{3+}-0.008,0.04,0.02,0.4$. As a control, a Steinberg's medium with concentration of $20 \%$ was used [5].

One sample of the apical sprouts of elodea was placed in each environment, plants were pre-selected according to the average indicators for the group of studied samplesmillisecond-delayed fluorescence when excited with high-intensity light. The test vessels were placed in a KV-3 cultivator at a temperature of $25{ }^{\circ} \mathrm{C}$. The metal content in the aqueous medium was controlled through mass-spectrometry.

In experiments with oil and oil products, only aqueous extracts of pollutants were used: "water-water oil extract", "water-water gasoline extract", "water-water diesel fuel extract". We also analyzed plants that were treated with solutions of salts and acids with a predetermined concentration for 20 minutes:

1 - elodea, processed dist. water - verification

2 - elodea treated with $0.5 \mathrm{M} \mathrm{CH}_{3} \mathrm{COONH}_{4}$

3 - elodea treated with $1 \mathrm{M} \mathrm{CH}_{3} \mathrm{COONH}_{4}$

4 - elodea treated with $0.01 \mathrm{M} \mathrm{Ni}_{2} \mathrm{Cl}_{2}$

5 - elodea treated with $0.01 \mathrm{M} \mathrm{Na}_{2}$ EDTA 
6 - elodea treated with $0.1 \mathrm{M} \mathrm{HNO}_{3}$

7 - elodea treated with $0.1 \mathrm{M} \mathrm{H}_{2} \mathrm{SO}_{4}$

8 - elodea treated with $0.1 \mathrm{M} \mathrm{HCl}$

The hydrocarbon composition in the prepared water samples was determined with a gas chromatograph and a Clarus 500 flame ionization detector (Perkin Elmer, USA). For this, a water sample of $50 \mathrm{ml}$ was poured into a measuring cylinder, each sample was acidified with a hydrochloric acid solution and $\mathrm{pH}=2,4.5 \mathrm{~g}$ of magnesium sulfate was added for every $50 \mathrm{ml}$ of a sample, and the sequestrant ratio was 1:1. As extractant, we used $\mathrm{n}$ hexane. Next, sequestration was carried out for one hour on a mechanical centrifuge.

\subsection{Methods}

\subsubsection{Fluorimeter "Photon-10"}

The registration of delayed chlorophyll fluorescence was performed using the computerized fluorimeter "Photon-10". This device is intended for registration of several delayed and variable fluorescence parameters of different plant objects (chloroplasts, algae, plant leaves, lichen). In this device, the patented method of rapid assessment of chemical substances is the phytotoxic effect, which based on the registration of the relational index in delayed chlorophyll fluorescence [6]. The variable fluorescence intensity measured upon excitation by light was both high and low intensity ( $R F h$ and $R F l$, respectively). The relative fluorescence indicator (RFI) is calculated on the basis of the data obtained as the ratio of high fluorescence to low florescence. This eliminates the dependence of the results on the area of leaf blades of plants. Registration of the parameters of RFI was carried out 1, 3, 7 days after placing the plants in the solution.

For ICP MS, the samples of plants were dried at a temperature not higher than $80{ }^{\circ} \mathrm{C}$ (to decrease the loss of highly volatile elements). After homogenization and obtaining an average sample, the weighted portions with the mass of $0.5 \mathrm{~g}$ were decomposed in a microwave oven Anton Paar GmbH (Perkin-Elmer) in a mixture of purified concentrated nitric and sulfuric acids at a temperature of $230-250{ }^{\circ} \mathrm{C}$. The elements in all the liquids were determined by the method of mass spectrometry with inductively coupled plasma (ICP MS) on a quadruple mass spectrometer Agilent 7500a (Agilent Technologies, USA). The samples were diluted 125 times with a solution of HNO $3(0.3 \mathrm{wt} . \%)$, with deionized water. Possible damage to the structures of the aquatic plant of elodea was determined through infrared Fourier spectrometry.

\subsubsection{Variable fluorescence}

Spectra in the range $4000-400 \mathrm{~cm}^{-1}$ were recorded on a Tensor 27 IR Fourier spectrometer (Bruker, Germany), and reflection spectra using an EasiDiff diffuse reflectance attachment (Bruker, Germany). Spectral information was processed using the OPUS 5.0 software package. A Nicolet 380 FTIR spectrometer (Thermo Electron Corporation, USA) was also used. In order to obtain IR absorption spectra, plant samples were prepared in the form of tablets in a potassium bromide matrix. Sample preparation conditions (mixing time with potassium bromide, molding pressure, vacuumizing) are the same. The concentration of the substance is constant and amounts to $6 \mathrm{mg}$ substance / $1000 \mathrm{mg} \mathrm{KBr}$. Shredded samples of plant fiber were subjected to analysis, after preliminary removal of cell and liquid and drying in an oven at a temperature of $50^{\circ} \mathrm{C}$. IR diffuse reflectance spectra of samples were taken directly from the surface of plant leaves, dried between glass slides. 


\subsubsection{Measuring metal cell wall bound fractions}

The method used to measure the cell wall bound fraction was modified after LozanoRodriguez et al. [7]. Four of the total 12 treatments were used, and the treatments chosen were the ones where metal addition during loading were the lowest and highest respectively, combined with the lowest and highest metal content during exposure. Shoots of E. canadensis were washed in distilled water and thereafter the stem and leaves were homogenized in an extraction buffer $(50 \mathrm{mM}$ HEPES, $500 \mathrm{mM}$ sucrose, $1.0 \mathrm{mM}$ DTT, $5.0 \mathrm{mM}$ ascorbic acid and $1.0 \%$ (w/v) Polyclar AT PVPP, $\mathrm{pH}$ adjusted to 7.5 with $\mathrm{NaOH}$ ) using Polytron ultra mixer. Cell wall fraction (fraction 1) was separated from the rest of the plant by sieving the homogenate through a nylon cloth with pore size $100 \mathrm{M}$, where cell wall fraction constituted the residue. The filtrate constituted the rest of the plant material (fraction 2). Fraction 1 was dried and thereafter fractions 1 and 2 were digested and analyzed for its metal content according to above-described method.

\subsubsection{Quality Assurance.}

Blanks and source of quality control standards were measured at every five samples to detect contamination and drift. The elemental concentrations of procedural blanks were generally $5 \%$ of the mean analyzed concentrations for all pollutants. Precision and accuracy of analysis were ensured through replicate analysis of the samples, acceptable percentage recoveries $(86.5 \% \pm 0.005$ to $96.4 \% \pm 0.003)$ were obtained from spiked digested vegetable samples.

\section{Results}

\subsection{Cell membrane resistance as an indicator of adaptive plant properties.}

Possible violations of cell membranes can serve as an indicator of the resistance of an aquatic plant to stress. Possible violations of cell membranes can serve as an indicator of the resistance of an aquatic plant to stress. The main method for this was selected by IR Fourier spectroscopy. IR absorption spectra and diffuse reflection spectra of the samples under study are similar to IR spectra of any plant material, in particular, cellulose. A spectroscopic study of such systems is based on a comparison of the spectral characteristics of the absorption bands of the functional groups of the control sample with the corresponding characteristics of these bands in the spectra of the processed samples. Let us consider the main absorption bands characteristic of the IR spectra of the studied samples.

In fig. 1 shows the IR absorption spectra and the diffuse reflectance spectra of the walls of the cell residues of plants from the control experiment (without introducing metal) and the experimental system with $\mathrm{Pb}^{+2}$. Intense bands are observed in the presented spectra.

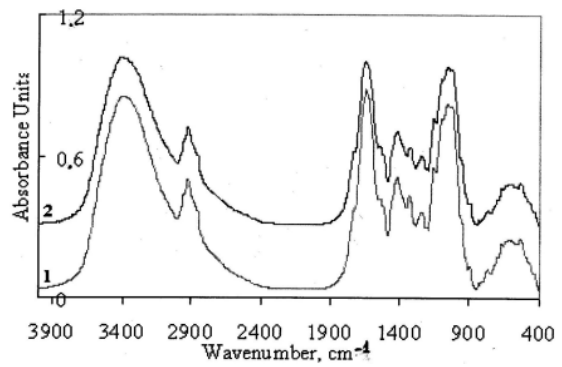

Fig. 1. IR spectra of samples of elodea: 1) control sample; 2) experimental sample 
As can be seen IR spectra had the absorption (p.p.) with maxima $\approx 3400$ and $1656 \mathrm{~cm}^{-}$ ${ }^{1}$. The intense wide absorption band in the region of $3700-2200 \mathrm{~cm}^{-1}$ (the main maximum is $\approx 3400 \mathrm{~cm}^{-1}$ ) is assigned to stretching vibrations of hydroxyl groups bound by hydrogen bonds $[8,9]$, and pp 1656 and $620 \mathrm{~cm}^{-1}$, respectively to plane deformation vibrations of $\mathrm{OH}$ groups $[8,9]$. The absorption bands in the regions of 3000-2800 and $1450-1370 \mathrm{~cm}^{-1}$ are caused, respectively, by stretching and deformation vibrations of aliphatic $-\mathrm{CH}_{3}$ and $-\mathrm{CH}_{2}$ groups $[8,9]$. The absorption band with a maximum of $1735 \mathrm{~cm}^{-1}$, as in $[8,9]$, is assigned to stretching vibrations of carbonyl groups. In addition, the analysis of the observed absorption bands in the region of 1000-1200 $\mathrm{cm}^{-1}$, together with the absorption at $1735 \mathrm{~cm}^{-}$ 1 , allows us to make an assumption about the presence of ketone-ether compounds in the structural components of the cell (cell walls).

The analysis of the presented curves indicates practically complete similarity of spectral patterns for all samples. This circumstance allows us to state that the treatment of plants with ammonium acetate does not affect the membrane (fiber) part of the plant. Changes, apparently, should be expected in the cell fluid. Although, it is unlikely that the acetate ion could easily penetrate a natural dialyzer, which, in our opinion, is represented by the membrane. And the second - the identity of the spectral patterns also indicates that the plants were washed from treatment solutions very carefully. A similar situation is observed in the case of comparing the spectra shown in Fig. 2.

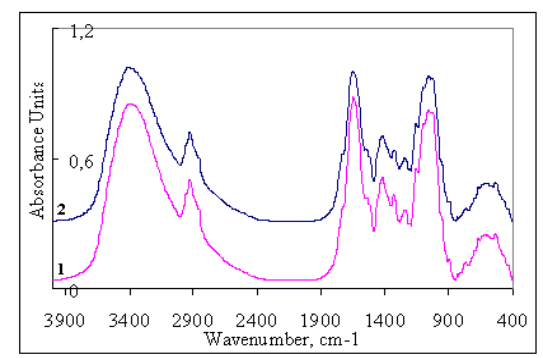

Fig. 2. IR spectra of fiber samples: curve 1 - control, curve 2 - sample treated with $0.01 \mathrm{M} \mathrm{NiCl2}$.

However, in this case, in our opinion, one should expect changes in the composition of the cell fluid only in terms of the amount of chlorine ion. It is hard to imagine that the nickel ion will pass into the cell.

Figure 3 shows the IR spectrum of the sample treated with a $\mathrm{Na}_{2}$ EDTA salt solution (curve 2) in comparison with the spectrum of the control sample (curve 1).

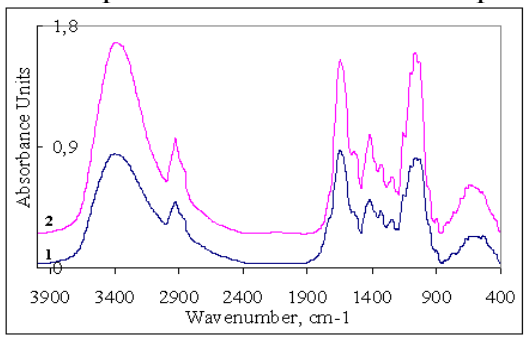

Fig. 3. IR spectra of fiber samples: curve 1 - sample 1, curve 2 - sample treated with $0.01 \mathrm{M}$ Na2EDTA.

The spectral patterns are identical, but the intensities of the observed absorption bands in the spectrum of sample treated $0.01 \mathrm{M} \mathrm{Na}_{2}$ EDTA are almost two times higher than in the case of the control sample. This fact can be explained by the assumption that treatment with a sequestrant, a solution of EDTA sodium salt, leads to the "leaching out" of the inorganic component of fiber. No traces of the reagent used for processing were detected in the spectra. 
In fig. 4 shows the IR spectra of samples treated with mineral acids (curves 1-3, respectively) in comparison with the spectrum of the control sample (curve 4).

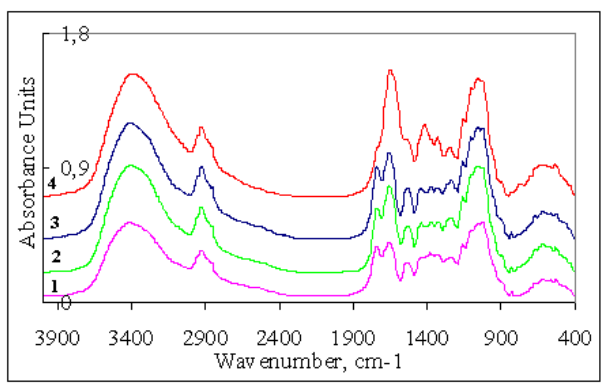

Fig. 4. IR spectra of fiber samples: curve 1 - sample treated with $0.1 \mathrm{M} \mathrm{HCl}$, curve 2 - sample treated with $0.1 \mathrm{M} \mathrm{H} 2 \mathrm{SO} 4$, curve 3 - sample treated with $0.1 \mathrm{M} \mathrm{HNO} 3$, curve 4 - control.

In the spectra of samples treated with acids (curves 1, 2, 3, Fig. 4) in p. $1740 \mathrm{~cm}^{-1}$, the intensity of which increases in the order of $\mathrm{HNO}_{3}>\mathrm{H}_{2} \mathrm{SO}_{4}>\mathrm{HCl}$, i.e., in proportion to the increase in the oxidizing ability of the acids used. This fact, in our opinion, is associated with partial oxidation of fiber with mineral acids. Thus, in contrast to the previously described leaf treatments with various chemicals, even short-term treatment of leaves with acids followed by their washing leads to partial destruction of fiber.

The diffuse reflectance spectra of the samples are shown in Fig. 5 and 6.

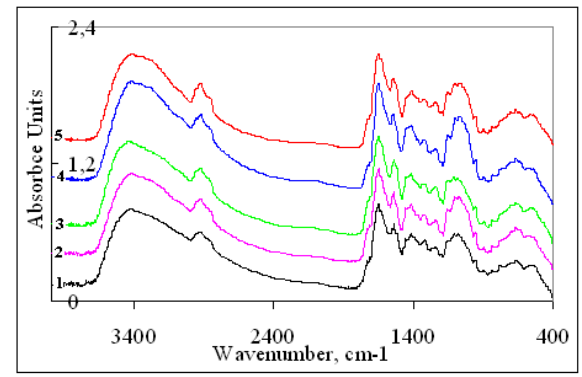

Fig. 5. IR spectra of diffuse reflection of fiber samples: 1 - control, 2 - sample treated with $0.01 \mathrm{M}$ $\mathrm{Ni2Cl} 2,3$ - sample treated with $0.5 \mathrm{M} \mathrm{CH} 3 \mathrm{COONH} 4,4$ - sample processed 1M CH3COONH4, 5 sample treated with $0.01 \mathrm{M} \mathrm{Na2EDTA.}$

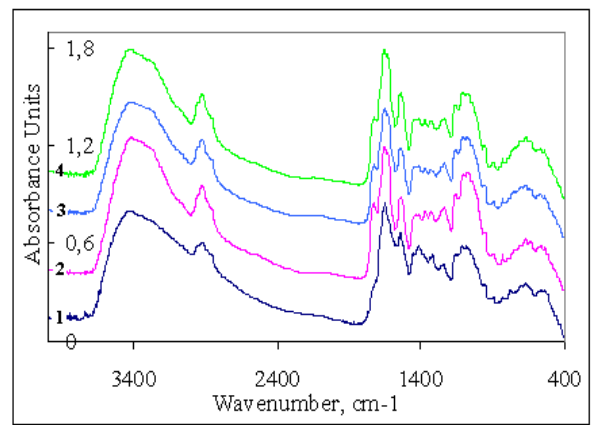

Fig. 6. IR spectra of diffuse reflection of fiber samples: 1 - control, 2 - sample treated with $0.1 \mathrm{M} \mathrm{HCl}$, 3 - sample treated with $0.1 \mathrm{M} \mathrm{H} 2 \mathrm{SO} 4,4$ - sample processed $0.1 \mathrm{M} \mathrm{HNO} 3$

The patterns of changes in the IR spectra of diffuse reflection are similar to those established previously for the IR absorption spectra. Gas chromatography studies have been 
carried out to determine certain functional groups of oil and oil products in the aquatic environment, which are among the most soluble components. Aqueous media were obtained by the interaction of oil, gasoline and diesel fuels with an aqueous medium. As shown in the studies, a large proportion are alkanes $(38.6 \%)$ and i-alkanes $(43.8 \%)$, more than two times less than cycloalkanes $(\sim 17 \%)$ and even less alkenes $(0.5 \%)$, hydrocarbons with $\mathrm{C} \geq 14(0.1 \%)$ and aromatics $(0.01 \%)$, of the total amount of hydrocarbons dissolved in water. When comparing the IR spectra of Elodea in control experiments and experiments with the addition of aqueous extracts of oil and oil products, a significant change in the chemical composition of the plant was noted (Fig. 7).

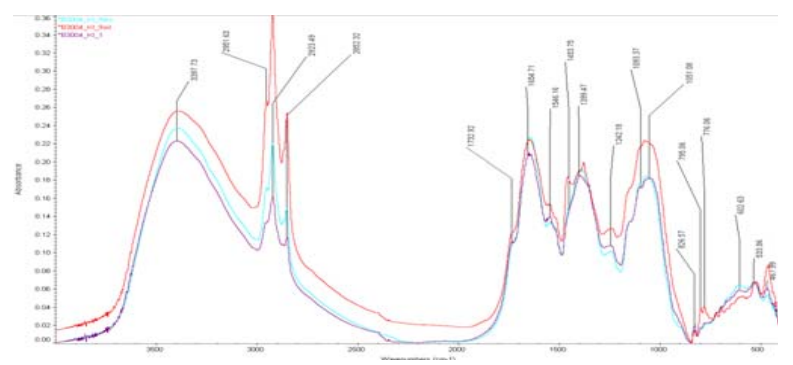

Fig. 7. IR spectra of elodea in experiments with aqueous extracts of oil, gasoline and diesel fuel.

So, for example, changes in vibrational frequencies in the bands: $1453.776,795 \mathrm{~cm}^{-1}$ indicate changes in the prevailing bonds (Fig. 7): methylene groups $\omega_{\mathrm{RCH}} \mathrm{Cl}-1240-1300$ $\mathrm{S}$; acid salts acetates: 1450-1600; ammonium salts (solid) $\left(\mathrm{NH}_{4}\right) \mathrm{A}^{-} \delta \mathrm{NH}_{4}^{+}-1390-1485 \mathrm{~S}$; borozol compound $\mathrm{BCH}_{3}$ 1405-1460; saturated aliphatic hydrocarbons: a) methyl groups, $\mathrm{ROCH}_{3}$; b) methylene groups $\mathrm{RCH}_{4} \mathrm{R}^{\prime} 1450-1475, \mathrm{RCH}_{2} \mathrm{NR}_{2}$ ' - 1445-1475; lactams (cis-Nsubstituted amides) 1440-1490; nitrosamines arrn-N $=\mathrm{O} v_{\mathrm{NO}}$ 1450-1500; primary aliphatic $\mathrm{RCH}_{2} \mathrm{NH}_{2} \omega_{\mathrm{NH} 2} 750-850 \mathrm{~S}$; thiacarbonyl compounds $v_{\mathrm{c}=\mathrm{s}} 740-1420 \mathrm{~V}$.

\subsection{Indications of delayed fluorescence as an indicator of plant viability.}

When choosing the concentration of the introduced metals, we took into account sanitary standards established in the Russian Federation for surface water bodies for economic purposes. The research results are shown in Fig. 8-10.

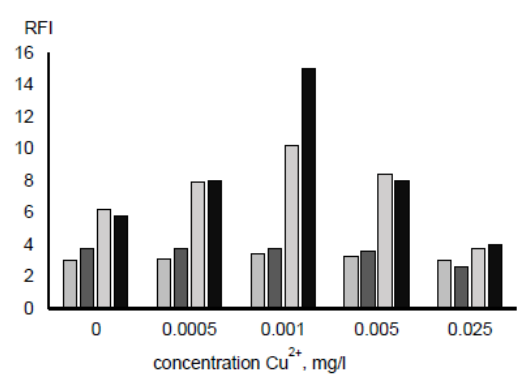

a)

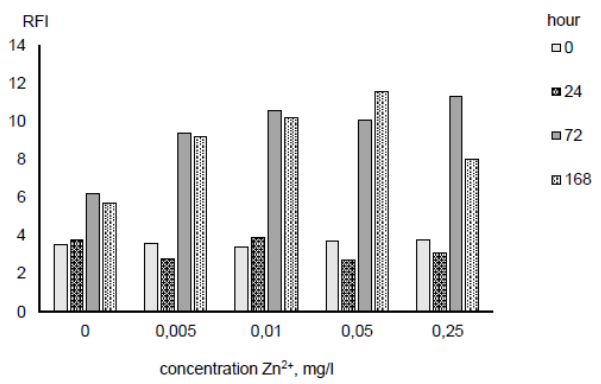

b)

Fig. 8. The effect of the concentration of copper ions (a) and zinc ions (b) on the magnitude of the RFI of elodea. 


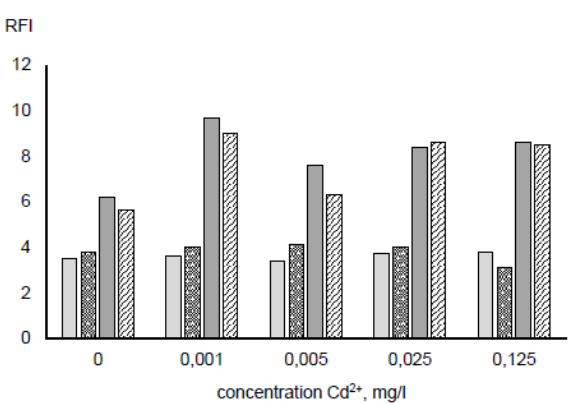

a)

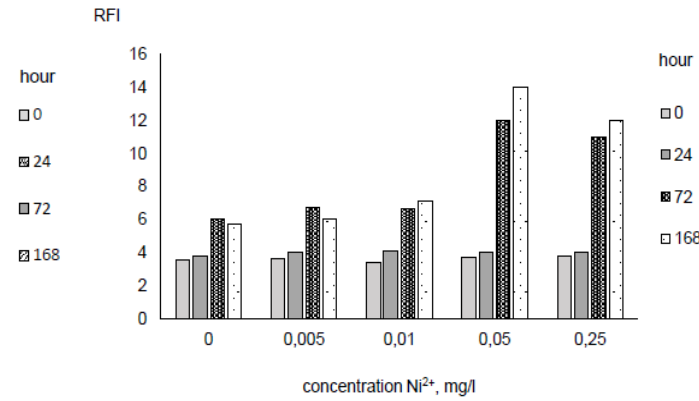

b)

Fig. 9. The effect of the concentration of cadmium ions (a) and nickel ions (b) on the magnitude of the RFI of elodea.

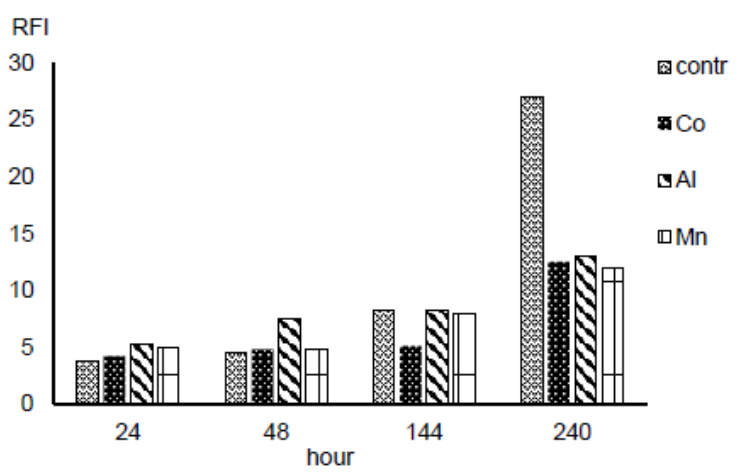

Fig. 10. The effect of heavy metal ions at a concentration metals on the RFI of elodea: Co $-0.01 \mathrm{mg} / \mathrm{l}$; $\mathrm{Mn}-0.01 \mathrm{mg} / \mathrm{l} ; \mathrm{Al}-0.04 \mathrm{mg} / \mathrm{l}$

In the presence of copper ions, a gradual increase occurs in the metal content in the walls of cell membranes. The higher the concentration of the substance, the faster its accumulation and subsequent elimination. The maximum accumulation occurs in the range from 1 to $25 \mu \mathrm{g} / 1$ in the early stages (3-7 days), and at $0.2 \mu \mathrm{g} / 1$ in the later ( 15 days). It should be especially noted that when $0.2 \mu \mathrm{g} / 1$ was added, the copper content in the cell membranes at the end of the experiment was higher than at 5 and $25 \mu \mathrm{g} / \mathrm{l}$. This can be explained by the fact that, at the lowest concentration, copper removal processes are minimal. Similar dependencies were revealed for other studied metals.

Data on the measurement of the relative delayed fluorescence in systems with oil, water extracts of oil and gasoline had had a predictable pattern.

Since the relative fluorescence index characterizes the activity of the photosynthetic apparatus, we can conclude that the elodea samples are best adapted in the water-oil system.

\section{The discussion of the results}

Environmental pollution from industrial emissions can be considered as a new environmental factor. Accepting this statement and assessing the resistance of plants to technogenic pollution as a new factor in the environmental-evolutionary plan, it should be recognized that in the arsenal of protective agents that ensure plant resistance, there are no specialized adaptations that ensure their successful growth under these conditions [10]. 
Thus, plants are used to reduce the negative effects of chemicals through mechanisms that have already been developed in the course of adaptation to other environmental factors.

In the example of the conducted studies, it can be noted that in plants, the structure of cell membranes changes, despite the fact that the level of exposure to the elodea was variable, both in strength (strong mineral acids) and in intensity (duration of exposure). Substances dissolved in the aquatic environment are an important abiotic stress for living organisms developing in this environment.

Like all other abiotic stresses, dissolved organic and inorganic compounds lead to biochemical and genetic changes [11].

An analysis of the literature indicates that when plants adapt to various adverse factors, similar structural and metabolic changes occur if the adverse factor does not go beyond the genetically determined adaptive capabilities of the body. Some plant species, in particular Canadian Elodea, exhibit a fairly high resistance to heavy metal compounds. So, Elodea remained viable at all studied concentrations.

The toxic effect, which is recorded by a decrease in fluorescence indices on 1-3 days after the addition of salts to the medium, decreases in 5-7 days of cultivation, which is accompanied by an increase in RFI. The introduction of cadmium, zinc and nickel ions at a concentration of up to $25 \mathrm{MPC}$ has a stimulating effect on fluorescence parameters, which is also especially pronounced on days 5-7. Apparently during this time going on the connection of processes that contribute to the adaptation of Canadian Elodea to high concentrations of the studied compounds.

It is known that metals such as zinc and copper are trace elements involved in the physiological processes of living plants, are part of the carriers of the electron transport chain of photosynthesis. The degree of accumulation of metals in plant organisms depends on many external (the concentration of the element in the environment and its shape existence, $\mathrm{pH}, \mathrm{E} \mathrm{h}$, seasonality and endogenous rhythms, light, temperature, salinity, currents, drains) and internal (species-specificity, physiological state of the organism, developmental stage, age, genetic control) factors.

In addition, depending on the environment and concentration, these metals cause serious toxic effects at all levels of development. Lead and cadmium are initially highly toxic metals. Despite this, the substances dissolved in the reservoir do not exist as a simple inorganic substance. A significant amount of organic and inorganic substances that interact with substances entering the water are already present in the surface water body. In this case, either even more toxic forms of occurrence are formed, or weakly or entirely nontoxic forms.

Phytochelatins have been identified in many aquatic plants and photosynthetic organisms, ranging from elodea, gymnosperms to monocots and dicots. Phytochelatins (PCs) are synthesized from glutathione (GSH) (in some cases, related compounds) by PC syntheses (PCS), and play a role in the distribution and accumulation of $\mathrm{Cd}$ and some other highly toxic metals like $\mathrm{Ag}, \mathrm{Hg}$, As. All abiotic stresses can accumulate excess ROS (reactive oxygen species) at certain stage of stress exposure leading to oxidative stress. However, ROS are not only toxic compounds, but sometimes play as important regulators for many biological processes in plants such as cell cycle, programmed cell death, hormone signaling, biotic and abiotic cell responses. As common consequences of abiotic stresses, osmotic stress and the ubiquitous oxidative stress have been extensively studied and offer more and more evidences for cross-talk at various steps or levels in the complicated network of abiotic stress signaling pathways. To cope with heavy metal stress and associated oxidative stress, metallothionein, a well-known metal chelator and also antioxidant would possibly be a good way out. Thus, the studied external influences on the aquatic plant did not go beyond the adaptive capabilities of the Elodea canadensis for almost all the studied model systems. And, therefore, the immersed macrophyte Elodea 
canadensis is tolerant to both organic (oil, gasoline, sodium acetate, EDTA) and inorganic (heavy metal salts, acids) abiogenic effects.

Online references will be linked to their original source, only if possible. To enable this linking extra care should be taken when preparing reference lists.

\section{Conclusion}

Elodea canadensis is a submerged aquatic plant of slower flowing rivers, native to North America. It has been intentionally introduced into areas outside of its native range as an ornamental aquarium species. This species has a wide ecological tolerance and grows relatively fast. It is a perennial, overwintering in the deeper water, and reproducing asexually. Elodea remained viable at all studied concentrations. The toxic effect, which is recorded by a decrease in fluorescence indices on 1-3 days after the addition of salts to the medium, decreases in 5-7 days of cultivation, which is accompanied by an increase in RFI. The immersed macrophyte Elodea canadensis is tolerant to both organic (oil, gasoline, sodium acetate, EDTA) and inorganic (heavy metal salts, acids) abiogenic effects. Online references will be linked to their original source, only if possible.

\section{References}

1. $\quad$ L.M. Hu, W.P. Hu, J.C. Deng, et al., Ecol. Eng., 36, 1725 -1732 (2010)

2. K. Sand-Jensen, C.L. Møller, Freshwater Biol., 59, 427-437 (2014)

3. H. Vereecken, J. Baetens, P. Viaene, et al., Hydrobiology, 570, 205-210 (2006)

4. M. H. Barrat-Segretain, A. Elger, Journal of Vegetation Science, 15(1), 109-114 (2004)

5. B.S. Mohan, B.B. Hosetti. Environ. Res., 81, 259-274 (1999)

6. Yu.S. Grigoriev, E.A. Furyaev, A.A. Andreev, Pat. 2069851 Russian Federation, MPK7 G 01 N 21/64, G 01 N 33/00. Publ. 11/27/96, Bull. No. 33. - 3 p.

7. E. Lozano-Rodriguez, L.E. Hernández, P. Bonay, R.O. Carpena-Ruiz. J Exp Bot., 48, 123-128 (1997)

8. H.A. Szymanski, R.E. Erickson, Infrared Band Handbook, (Plenum Press, New York, 1970)

9. R.A. Nyquist, C.L. Putzig, M.A. Leugers, Handbook of Infrared and Raman Spectra of Inorganic Compounds and Organic Salts (Academic Press, San Diego, CA, 1997)

10. I.Yu. Usmanov, Z.F. Rakhmankulova, A.Yu. Kulagin. Ecological plant physiology (Logos, Moscow, 2001)

11. Peter M.A. Toivonen, David A. Brummell, Postharvest Biology and Technology, 48, $1-14$ (2008) 